\title{
Déclaration obligatoire de la rougeole en Suisse: forte augmentation du nombre de cas au printemps 2003
}

\author{
J.-L. Richard ${ }^{a}$, K. Boubaker ${ }^{b}$, M. Doutaz ${ }^{c}$, G. Schubiger
}

\section{Résumé}

Une importante épidémie de rougeole sévit en Suisse depuis le début de l'année 2003. Le nombre de cas rapportés de janvier à début juin dans le cadre de la déclaration obligatoire se monte à 464. Le nombre réel de cas est plus élevé, car tous les malades ne consultent pas ni ne sont déclarés. Les cantons les plus touchés sont Schwyz, le Valais, Fribourg, Zurich et Genève. Septante-neuf pour cent des cas avaient moins de 16 ans. Plus de $90 \%$ des cas n'étaient pas vaccinés. Cinquante et une complications et 26 hospitalisations ont été rapportées. A titre d'exemple, deux brèves descriptions de complications sont fournies. Une couverture vaccinale encore insuffisante permet la circulation du virus de la rougeole en Suisse et la survenue périodique d'épidémies.

\section{Introduction}

Maladie particulièrement infectieuse, la rougeole frappait la plupart des enfants de Suisse avant l'introduction de la vaccination en 1976. Parfois considérée - à tort - comme bénigne, cette maladie caractérisée par un exanthème généralisé et de fortes fièvres souvent accompagnés de toux, de rhinite, de conjonctivite et de taches de Koplik, peut entraîner des complications graves voire létales. Avec la généralisation de la vaccination contre la rougeole puis ROR (rougeole, oreillons et rubéole), le nombre de cas de rougeole a fortement diminué en Suisse et l'on est passé d'une transmission épidémique caractérisée par une circulation permanente du virus, avec des pics épidémiques tous les 2 à 4 ans [1], à une transmission sporadique avec des cas isolés et, plus rarement, des flambées locales. L'insuffisance de la couverture vaccinale contre la rougeole permet toutefois encore à des épidé- mies plus importantes de se développer périodiquement, comme c'est le cas depuis le début de cette année.

L'objectif de cet article est de décrire l'épidémie de rougeole du printemps 2003, en s'appuyant sur les données issues de la déclaration obligatoire. Nous insisterons en particulier sur ses causes et ses conséquences en termes de complications, notamment à travers deux brèves descriptions de cas: une méningo-encéphalite rougeoleuse et une pneumonie secondaire à une rougeole.

\section{Matériel et méthodes}

Depuis 1999, la déclaration individuelle par les médecins des cas cliniques de rougeole est obligatoire. Tous les cas présentant une fièvre, un exanthème maculo-papuleux et une toux, une rhinite ou une conjonctivite sont à déclarer au médecin cantonal. Depuis 1999 également, la déclaration individuelle des cas confirmés de rougeole par les laboratoires a remplacé l'ancienne déclaration collective. Les formulaires de déclaration initiale et complémentaire à remplir par les médecins et ceux pour les laboratoires sont fournis par les médecins cantonaux ou téléchargeables sur le site Internet de l'Office fédéral de la santé publique (OFSP) (www. bag.admin.ch/infreporting/forms/f/index.htm). Nous avons analysé les cas déclarés à l'OFSP survenus de début janvier à début juin 2003 (semaines 1 à 22).

Le réseau des médecins Sentinella suit également la rougeole depuis 1986. Sur une base volontaire, environ 250 médecins de premier recours déclarent chaque semaine à l'OFSP les cas cliniques d'un certain nombre de maladies, dont la rougeole. En raison de la sensibilité et de la spécificité différentes des deux systèmes, nous n'avons pas sommé les deux casuistiques.
E-mail:

jean-luc.richard@bag.admin.ch 
Figure 1

Nombre de cas de rougeole déclarés par semaine en Suisse, en 2003.

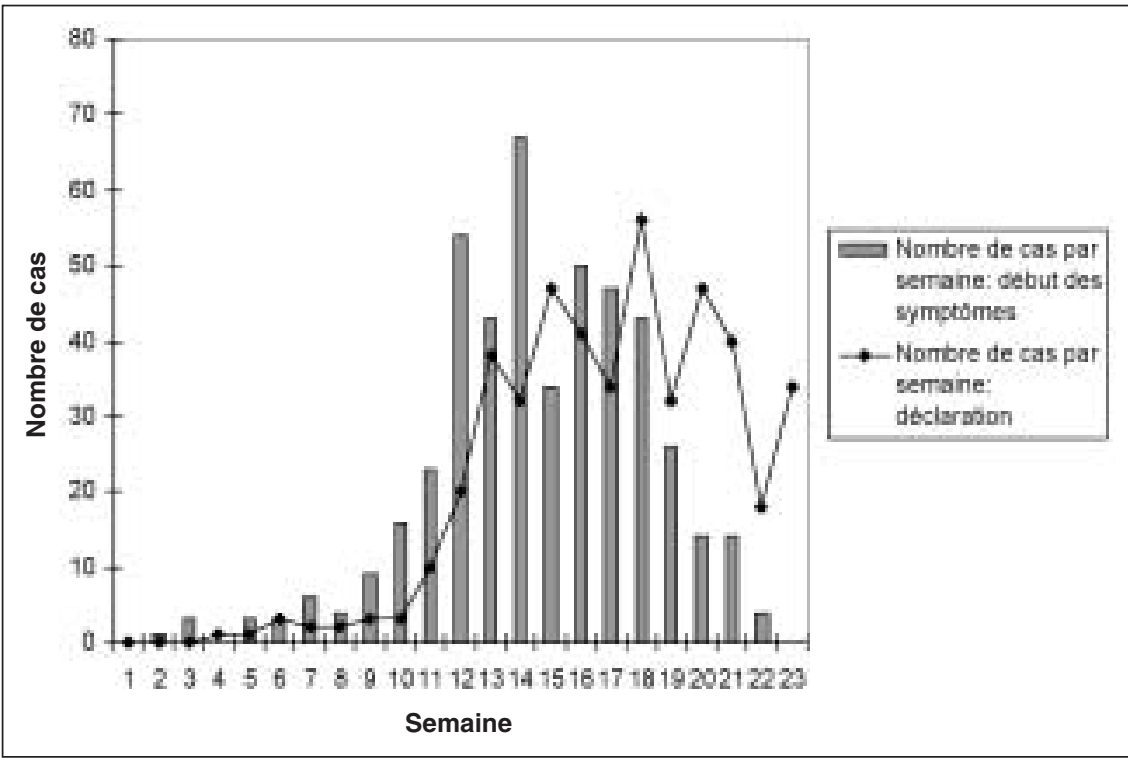

Figure 2

Répartition des cas de rougeole déclarés en Suisse, en 2003 (semaines 1 à 22).

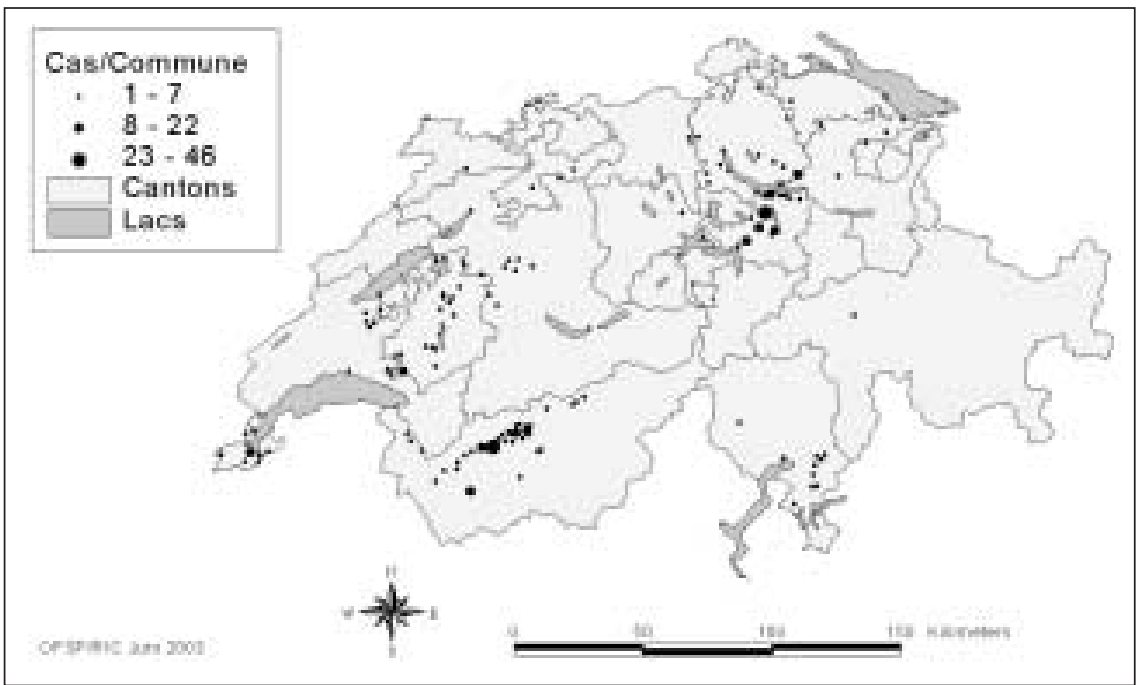

\section{Résultats}

\section{Nombre de cas déclarés}

Les cinq premiers mois de l'année 2003, 464 cas de rougeole ont été déclarés. Nous disposons d'une déclaration complémentaire des médecins chez 436 (94,0\%) d'entre eux. La courbe épidémique montre une forte augmentation à partir de la semaine 7, pour atteindre 67 cas durant la semaine 14 (fig. 1). Le pic de l'épidémie semble actuellement dépassé.
L'épidémie de rougeole sévissant actuellement se caractérise par la multiplication de flambées locales ou régionales et donc par une grande hétérogénéité spatiale à l'échelle de la Suisse (fig. 2). A eux seuls, cinq cantons ont enregistré $85 \%$ des cas: Valais (162 cas, 35\%), Schwyz (98 cas, $21 \%$ ), Fribourg (53 cas, $11 \%$ ), Zurich (42 cas, $9 \%$ ) et Genève (39 cas, $8 \%$ ). Au total, 18 cantons ont déclaré des cas. Comme le montre la figure 2 , on observe des groupements de cas à l'intérieur même des cantons les plus touchés.

Presque autant de femmes (221/456, 47,6\%) que d'hommes ont été déclarées. L'âge médian des cas était de 10,3 ans. Trente pour cent des cas avaient 5 à 9 ans, 26\% 10 à 14 ans, 17\% 15 à 19 ans, $17 \% 1$ à 4 ans, $8 \% 20$ ans et plus et $2 \%$ moins de 1 an (fig. 3). La structure par âge était différente pour les trois principales flambées de cas. Dans le canton de Schwyz, la rougeole a surtout touché les enfants de 5 à 9 ans $(46 \%$ du total des cas du canton). En Valais, elle a surtout frappé les grands enfants et les adolescents (70\% des cas chez les 10 à 19 ans), alors que dans le canton de Fribourg elle a surtout concerné les 5 à 14 ans (75\% des cas). Ces différences reflètent largement le type du foyer initial de deux de ces flambées: une école primaire à Fribourg et un gymnase en Valais.

Parmi 395 cas (85\%) pour lesquels le statut vaccinal est connu, 38 (9,6\%) étaient vaccinés contre la rougeole, dont 34 (91\%) avec une seule dose, trois avec deux doses et un avec un nombre inconnu de doses. Dix cas vaccinés avec une seule dose ont été confirmés par une analyse de laboratoire, ainsi qu'un cas ayant reçu deux doses. Si l'on excepte les enfants de moins de 1 an et les personnes de 20 ans et plus, la proportion des cas vaccinés est relativement stable en fonction de l'âge (fig. 3).

Un examen de laboratoire a été effectué pour 136 (29\%) cas cliniques de rougeole, permettant de confirmer 125 cas, soit $27 \%$ du total des cas déclarés. Le génotype du virus a pu être déterminé pour 16 cas: sept à Schwyz, sept en Valais et deux à Genève. Les virus circulant à Schwyz et à Genève appartiennent au génotype D8, ceux du Valais au génotype D5. Le premier est endémique en Inde, au Népal et en Ethiopie; le second au Japon et en Thaïlande [2]. Ces données de laboratoire confirment l'origine asiatique de la flambée de cas enregistrée dans le canton de Schwyz. Le cas index est un enfant de ce canton ayant eu une rougeole peu de temps après son retour de vacances de fin d'année en Inde. Il l'a transmise à des élèves de l'école privée qu'il fréquente. Les élèves de cette école sont ensuite allés en semaine de ski dans une autre région de 
Figure 3

Nombre de cas de rougeole déclarés en 2003 à l'Office fédéral de la santé publique par groupe d'âge et statut vaccinal (semaines 1 à 22).

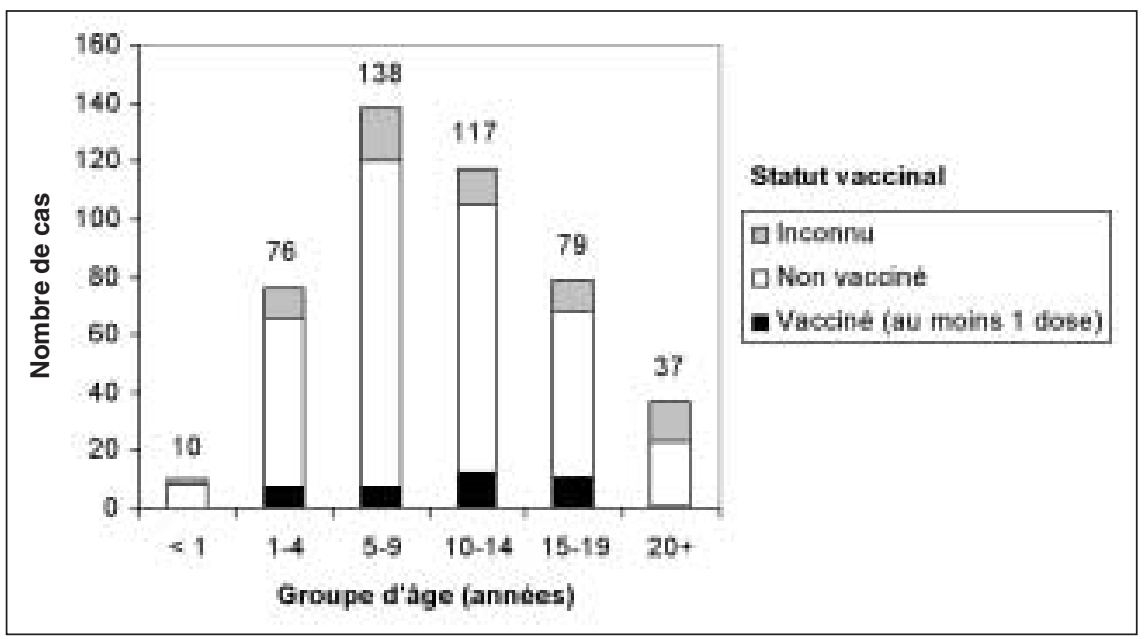

Schwyz, ce qui a contribué à diffuser la maladie dans le canton. Aucun lien épidémiologique entre les cas schwytzois et genevois n'a pu être mis en évidence.

\section{Complications}

Pour les 22 premières semaines de l'année 2003, $51(11,0 \%)$ des 464 cas déclarés ont présenté une complication. Il s'agissait de trois encéphalites, 14 pneumonies, trois suspicions de pneumonie, 11 otites, un delirium, une myocardite, un cas présentant des convulsions toniques généralisées, une gingivostomatite, une hépatite avec suspicion d'appendicite, une otite avec hépatite et 14 hospitalisations sans mention de complication. Vingt-six $(5,6 \%)$ personnes ont été hospitalisées, dont une pendant près de 6 semaines pour une encéphalite rougeoleuse. Aucun décès n'a été signalé.

Le risque de complications augmente avec l'âge. Il passe de 6,6\% pour les enfants de 1 à 4 ans à $24,3 \%$ pour les adultes de 20 ans et plus. Trente-deux $(8,9 \%)$ enfants de moins de 16 ans ont eu une complication contre $18(18,8 \%)$ adultes $(\mathrm{p}=0,006)$. Les trois cas avec encéphalite avaient respectivement 10, 10 et 16 ans. Deux $(5,3 \%)$ cas vaccinés ont eu une complication, contre $43(12 \%)$ cas non vaccinés et $6(8,7 \%)$ cas avec un statut vaccinal inconnu (différence statistiquement non significative).

\section{Description de cas 1: Méningo-encéphalite rougeoleuse chez un adolescent}

Il s'agit d'un étudiant de 17 ans, en bonne santé habituelle, sans antécédents particuliers, non vacciné contre ROR, hospitalisé en urgence le
25 mars 2003 pour une perte de connaissance avec mouvements tonico-cloniques.

L'anamnèse, donnée par la famille, révèle un état fébrile depuis 7 jours accompagné de céphalées, myalgies, conjonctivite bilatérale avec rhinite claire et lésions cutanées du corps entier depuis 4 jours. Les lésions sont apparues sur le visage pour s'étendre rapidement au tronc puis aux quatre membres. Alors que l'évolution semble spontanément favorable, le patient présente une perte de connaissance avec mouvements tonico-cloniques. Notion d'un contage avec un cas probable de rougeole environ 10 jours avant le début des symptômes.

A l'admission, patient en mauvais état général, inconscient, afébrile à $37^{\circ} \mathrm{C}$, hypertendu à $170 / 70 \mathrm{mmHg}$ et bradycardie sinusale à 45 pls/ min. Exanthème maculo-papuleux avec pétéchies, prédominant au visage avec tuméfaction des paupières et sur le tronc. A l'examen neurologique on note un coma, des signes cortico-bulbaires et cortico-spinaux bilatéraux avec hyperréflexie, spasticité et Babinski bilatéraux. Le reste de l'examen clinique est dans les limites de la norme. Parmi les examens para-cliniques on relève des IgM et des IgG positives contre le virus de la rougeole et une thrombopénie modérée. L'IRM du 26 mars 2003 montre une pansinusite, celle du 31 mars 2003 une légère hyperintensité du signal pariéto-occipitale bilatérale diffuse de la substance blanche compatible avec une encéphalite.

Sur la base des examens cliniques et para-cliniques, le diagnostic de méningo-encéphalite rougeoleuse est retenu. Sous traitement symptomatique et soutien respiratoire, l'évolution est lentement favorable. Sans sédation, réveil progressif permettant l'extubation le 28 mars 2003. A ce stade, l'examen neurologique montre un score de Glasgow (GCS) à 15, une dysmétrie aux tests cérébelleux et la persistance d'une légère désorientation. L'évolution est favorable. A la sortie, on note, sur le plan clinique, la persistance d'un léger ralentissement psychomoteur, d'un réflexe palmo-mentonnier et de troubles oculomoteurs ainsi que d'une brady-dysrythmie intermittente fronto-temporale droite avec activité de fond normale à l'EEG. Un bilan neuropsychologique effectué après la sortie montre une récupération complète.

\section{Description de cas 2: Complication pulmonaire sévère secondaire à une rougeole chez un enfant}

Un garçon âgé de 10 ans, sans antécédents de vaccination ROR, est hospitalisé pour une tachypnée, une dyspnée et une toux avec un état 
Figure 4

Patient intubé avec voie veineuse centrale à droite et sonde gastrique. Infiltrat pulmonaire bilatéral interstitiel important avec opacités macro-nodulaires, partiellement confluent, plus marqué dans la région centrale du lobe supérieur gauche, dans la partie paramédiastinale du lobe supérieur droit et dans les parties moyennes des deux lobes inférieurs. Petit épanchement pleural à droite, avec émoussement de l'angle costophrénique. Silhouette cardiaque de taille normale sans signes de décompensation.

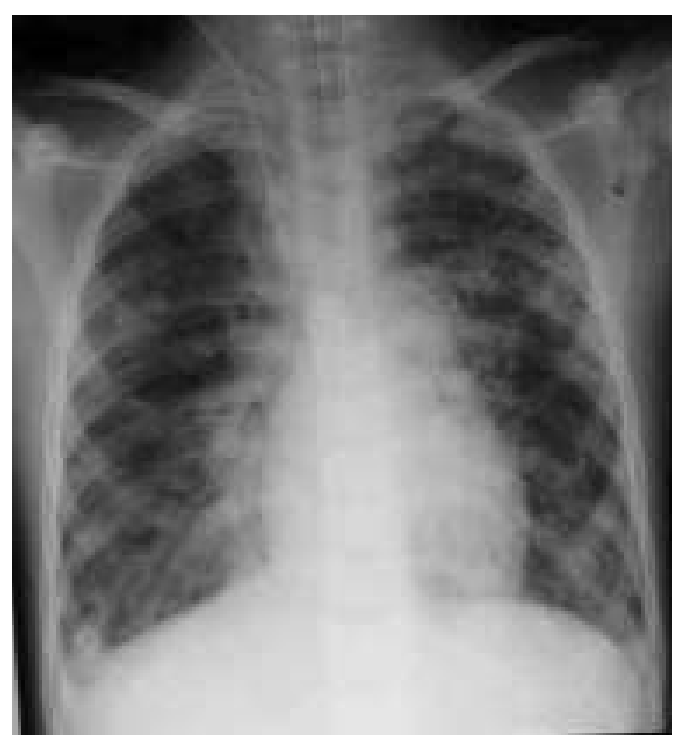

hautement fébrile 14 jours après le début d'une rougeole classique et après disparition de l'exanthème. Un traitement de vitamine A est instauré de même qu'une antibiothérapie, en raison d'arguments de laboratoire clairement en faveur d'une surinfection bactérienne. Le jour suivant, le patient doit être intubé et ventilé en raison d'une décompensation respiratoire imminente. La radiographie du thorax montre un infiltrat pulmonaire interstitiel avec opacités diffuses en progression (fig. 4). Aucune bactérie n'a pu être mise en évidence. Après 9 jours de traitement intensif, avec des besoins en oxygène jusqu'à $100 \%$ ainsi qu'un soutien par vasopresseurs, le patient peut être extubé. Il peut rentrer à domicile après 23 jours d'hospitalisation. Au vu de l'évolution clinique, le diagnostic retenu est celui de pneumonie morbilleuse menaçant la vie, avec possible surinfection bactérienne.

\section{Discussion}

Entre les semaines 1 et 22 de l'année 2003, la Suisse a enregistré une forte augmentation du nombre de nouveaux cas de rougeole déclarés à l'OFSP. Le nombre de cas notifiés dont les symptômes ont commencé durant les cinq premiers mois de l'année n'était en effet que de 9, 27 et
27, respectivement en 2000, 2001 et 2002, pour 464 en 2003. Ces derniers ne constituent cependant qu'une fraction du total des cas de rougeole survenus au début de cette année en Suisse. D'une part, tous les cas ne débouchent pas sur une consultation, tout particulièrement lors d'importantes flambées de cas; d'autre part, tous les cas vus en consultation ne sont pas déclarés, notamment parce qu'une partie des médecins ignorent que la rougeole est à déclaration obligatoire depuis 1999. Dans des cas extrêmes, la combinaison de ces deux facteurs peut entraîner une sous-estimation considérable de la situation réelle. Par exemple, sur les 42 cas survenus dans une école schwytzoise, seuls deux ont été portés à la connaissance du médecin cantonal et de l'OFSP au moyen des formulaires de déclaration. Selon une étude italienne, la déclaration obligatoire de la rougeole pour les enfants fournit près de 4 fois moins de cas que les extrapolations effectuées à partir des déclarations des pédiatres sentinelles [3]. Pour l'épidémie de rougeole de 1999 et 2000 aux Pays-Bas, on a estimé que le nombre réel des cas était probablement 10 fois supérieur aux 3292 cas rapportés [4].

L'introduction d'une vaccination chez les jeunes enfants avec une couverture insuffisante tend à pousser vers le haut l'âge moyen des cas. Avec $21 \%$ des cas âgés de 16 ans ou plus et un âge médian de 10,3 ans, l'âge des cas durant l'épidémie du printemps 2003 est relativement élevé, comparé à la situation sans vaccination. Les données disponibles dans le cadre de la déclaration obligatoire sont trop récentes et pas assez étendues (1999-2003) pour observer une éventuelle élévation de l'âge des cas de rougeole. Disponibles depuis 1986, les données Sentinella ne montrent toutefois aucune tendance en ce sens.

Dans la plupart des cas, les patients atteints de rougeole guérissent sans complications après 7 à 10 jours [5]. En moyenne, un cas sur sept à dix souffre cependant d'une complication pouvant, environ une fois sur 1000 à 2000 dans les pays industrialisés, entraîner le décès [6]. Les complications les plus redoutées sont les encéphalites et les pneumonies. Les premières surviennent en moyenne dans un cas sur 500 à 5000, les secondes dans un cas sur 17 à 100 [6]. Le taux de complications observé durant la présente épidémie correspond aux valeurs de la littérature. De telles comparaisons restent cependant délicates pour des données issues de la surveillance passive, qui souffre probablement d'un biais de déclaration différent pour les cas simples et les cas avec complications. La fréquence des encéphalites paraît par contre très élevée, avec une encéphalite pour 155 cas de 
rougeole déclarés. Une quatrième encéphalite a de plus été déclarée par un médecin Sentinella. La question reste ouverte s'il faut y voir un hasard, l'indice d'une sous-déclaration massive des cas sans complications graves, qui affaiblirait le dénominateur, ou encore une virulence particulière des virus en circulation. Malgré les différents types viraux prévalents, on n'observe aucun signe d'une diminution d'efficacité des vaccins anti-rougeoleux contre la maladie. Non seulement la vaccination protège efficacement contre la rougeole, mais nos données semblent montrer que, en cas de maladie, elle assure aussi une certaine protection contre les complications de la rougeole. Les données de surveillance passive ne sont cependant pas idéales pour l'étude des taux de complications et nos résultats ne sont pas statistiquement significatifs. Conformément à ce que rapporte la littérature, nous avons par contre pu mettre en évidence une augmentation du taux de complications en fonction de l'âge [7].

L'OFSP a recommandé dès 1976 la vaccination contre la rougeole au moyen d'une dose unique à 12 mois d'un vaccin monovalent. La vaccination avec le vaccin combiné ROR est recommandée depuis 1985 pour les enfants de 15 à 24 mois, avec rattrapage à 12 à 15 ans. Une seconde dose a été introduite en 1996 pour les enfants de 4 à 7 ans [8]. En 2001, l'âge d'administration de la première dose a été ramené de 15 à 12 mois, celle de la deuxième dose à 15 à 24 mois [9]. Des rattrapages sont prévus en début et en fin de scolarité ainsi qu'en tout temps pour les jeunes adultes. Malgré ces recommandations et des campagnes de promotion des vaccinations de routine initiées en 1987 et en 2002, la couverture vaccinale de la rougeole est restée stable en Suisse durant la décennie écoulée, aux alentours de $80 \%$ pour les jeunes enfants $[10,11]$. En
2000-2002, la couverture vaccinale des enfants de 24 à 35 mois était de $81 \%$. Pour au moins une dose, elle s'élevait à $89 \%$ chez les enfants de 5 à 7 ans et à $94 \%$ chez les adolescents de 16 ans [12]. Or pour interrompre la circulation du virus et éliminer la rougeole d'ici 2010 selon l'objectif de l'OMS pour l'Europe [13], il est nécessaire qu'au moins $95 \%$ des enfants soient vaccinés deux fois.

Les déclarations de cas provenant du Valais suggèrent que l'absence de vaccination résulte largement du choix d'une minorité de parents, dont les enfants sont suivis par des médecins qui n'encouragent pas la vaccination contre les ROR, voire lui sont opposés. Les cas déclarés tendent en effet à s'y concentrer autour de quelques médecins alternatifs. De plus, le foyer de la flambée schwytzoise était une école privée offrant une pédagogie alternative. Quarante-deux des 46 élèves y ont contracté la rougeole en quelques semaines.

La couverture vaccinale insuffisante, en particulier chez les jeunes enfants, et l'existence de poches de population non immune permettent périodiquement la survenue d'épidémies de rougeole en Suisse. Depuis sa création en 1986, le réseau Sentinella avait déjà détecté des épidémies en 1987 et en 1997, avec un nombre de cas estimé pour la Suisse de 10500 et 6400 respectivement [14]. Une intensification des efforts en matière de vaccination ROR est nécessaire pour éliminer la rougeole de Suisse, comme c'est le cas en Finlande depuis 1996 [15].

\section{Remerciements}

Les auteurs remercient Pierre-Alain Raeber, Hanspeter Zimmermann, Catherine Bourquin, Daniel Koch et Hans-Peter Roost de l'OFSP pour leurs commentaires critiques. 


\section{Références}

1 Gabutti G, Rota MC, Salmaso S, Bruzzone BM, Bella A, Crovari P and the Serological Study Group. Epidemiology of measles, mumps and rubella in Italy. Epidemiol Infect 2002; 129:543-50.

2 Rota PA, Bellini WJ. Update on the global distribution of genotypes of wild type measles viruses. J Infect Dis 2003;187(Suppl 1):S270-6.

3 Ciofi degli Atti ML, Salmaso S, Bella A, Arigliani R, Gangemi M, Chiamenti G, et al. Pediatric sentinel surveillance of vaccine-preventable diseases in Italy. Pediatr Infect Dis J 2002;21:763-8.

4 Van den Hof S, Conyn-van Spaendonck MAE, van Steenbergen JE. Measles epidemic in the Netherlands, 1999-2000. J Infect Dis 2002; 186:1483-6.

5 Gershon AA. Measles virus (rubeola). In: Mandell GL, Bennett JE, Dolin R (eds.). Principles and practice of infectious diseases. $5^{\text {th }}$ edition. Philadelphia: Churchill Livingstone; 2000. p. 1801-9.

6 Office fédéral de la santé publique et Commission suisse pour les vaccinations. Prévention de la rougeole, des oreillons et de la rubéole. In: Office fédéral de la santé publique (éd.). Maladies infectieuses: Diagnostic et prévention. Berne: Office fédéral de la santé publique; 2003. Supplément XII. (sous presse).

7 Miller DL. Frequency of complications of measles. Br Med J 1963;11:75-8.

8 Office fédéral de la santé publique et Commission suisse pour les vaccinations. Plan de vaccination de routine. In: Office fédéral de la santé publique (éd.). Maladies infectieuses: Diagnostic et prévention. Berne: Office fédéral de la santé publique; 1996. Supplément VIII.
9 Office fédéral de la santé publique et Commission suisse pour les vaccinations. Plan de vaccination de routine. In: Office fédéral de la santé publique (éd.). Maladies infectieuses: Diagnostic et prévention. Berne: Office fédéral de la santé publique; 2001. Supplément VIII.

10 Minder C, Steffen R. Vaccination des enfants en bas âge: Une enquête représentative sur la couverture vaccinale en Suisse 1991. Bull BAG/OFSP 1992;32:504-7.

11 Office fédéral de la santé publique. Vaccination des enfants en bas âge: Une enquête représentative sur la couverture vaccinale en Suisse 1998. Bull BAG/OFSP 1999;20:356-61.

12 Communication personnelle Phung Lang, Zurich.

13 Spika JS, Wassilak S, Pebody R, Lipskaya G, Deshevoi S, Güris D, et al. Measles and rubella in the World Health Organization European Region: diversity creates challenges. J Infect Dis 2003;187 (Suppl 1):S191-7.

14 Paget WJ, Zimmermann H, Vorkauf H, Groupe de travail Sentinella. Epidémie de rougeole en Suisse en 1997: conséquences sur l'élimination de la rougeole à l'horizon 2007. Eurosurveillance 2000;5:17-20.

15 Office fédéral de la santé publique. La Finlande a éliminé la rougeole. Bull BAG/OFSP 2003; 16:278. 